\title{
CDA Generation and Integration for Health Information Exchange based on Cloud Computing System
}

\author{
Shubham P. Lekule \\ Maharashtra Institute of Technology \\ Aurangabad
}

\author{
Saurabh H. Deshmukh, PhD \\ Maharashtra Institute of Technology \\ Aurangabad
}

\begin{abstract}
Successful readying of Electronic Health Record helps improve patient safety and quality of care, but it is the necessity of ability between Health data Exchange at fully totally different hospitals. The Clinical Document design (CDA) developed by HL7 may well be a core document traditional to verify such ability, and propagation of this document format is significant for ability. sadly, hospitals unit reluctant to adopt sensible HIS due to its activity worth apart from throughout a few countries. a haul arises even once extra hospitals begin victimization the CDA document format as a results of the information scattered in various documents unit taxing to manage. throughout this paper, we've a bent to explain our CDA document generation and integration Open API service supported cloud computing, through that hospitals unit enabled to handily generate CDA documents while not having to shop for proprietary computer code. Our CDA document integration system integrates multiple CDA documents per patient into one CDA document and physicians and patients can browse the clinical information in written record order. Our system of CDA document generation and integration depends on cloud computing and additionally the service is obtainable in Open API. Developers victimisation fully totally different platforms therefore can use our system to spice up ability.
\end{abstract}

\section{Keywords}

CDA Generation, Cloud Computing

\section{INTRODUCTION}

Electronic Health Record (EHR) is longitudinal assortment of electronic health data for and concerning persons, wherever health data is outlined as data touching on the health of a personal or health care provided to a personal and it will support of economical processes for health healthcare delivery. CDA (Clinical Document Architecture) by Health Level Seven could be a major normal for clinical documents . CDA could be a document markup normal that specifies the structure and linguistics of 'clinical documents' for the aim of exchange. the primary version of CDA was developed in two001 and unleash 2 came enter 2005 . several CDA-based comes are with success completed in several countries. Active works square measure being done on up linguistics ability supported open HER. to confirm ability of pelt along, the amount of HIS that supports CDA must be sufficiently giant. However, the structure of CDA is extremely complicated and therefore the production of correct CDA document is difficult to realize while not deep understanding of the CDA normal and enough expertise with it. additionally, the HIS development platforms for hospitals vary thus greatly that generation of CDA documents in every hospital invariably needs a separate CDA generation system. Also, there's a resistance towards new systems unless it's completely necessary for provision of care. As a result, the adoption rate of EHR is extremely low aside from a couple of handful countries like New Seeland or Australia . The US Government runs the significant Use Program to enhance potency in attention and patient safety. This program was launched as a section of incentives to boost the EHR adoption rate for EHR adopting hospitals . Even inside a town, a patient might head to totally different hospitals, and the personal medical records ar distributed across every hospital. Medical errors may be avoided if the connected medical data of the patient may be retrieved properly and with efficiency. thus the effective communication and availability of medical data among hospitals is associate degree important issue. In recent years, there are plenty of researches on totally different architectures for sharing medical records supported web technologies [1-3]. so as to reuse the sharable data, several medical standards ar proposed for the sleek exchange of electronic medical records following the particular standards. Health Level Seven (HL7) organization has worked hard to produce a comprehensive framework for the exchange and sharing of clinical data (such as discharge summaries and progress notes). Clinical Document Architecture (CDA), Release 1, became associate degree yankee National Standards Institute (ANSI) approved HL7 standard in two000 and CDA unharness 2 in 2005 [4]. The HL7 standard clearly defines the design of the changed data. According to CDA R2, CDA documents use the Extensible nomenclature (XML) format [5] for a good variety of applications. XML could be a versatile text format that's easy to use over the web and thus several applications are antecedently printed with it..

\section{RELATED WORK}

Many Clinical Document design (CDA) referrals and reply documents are accumulated for patients since the readying of the Health info Exchange System (HIES) in Choson. Clinical information were scattered in several CDA documents and this took an excessive amount of time for physicians to browse. Physicians in Korea pay solely restricted time per patient as insurances in Choson follow a fee-for-service model. Therefore, physicians weren't allowed comfortable time for creating edical selections, and follow-up care service was hindered. to handle this, we tend to developed CDA Integration model (CIT) and CDA Integration system (CIS) for the HIES. The clinical things enclosed in CIT were outlined reflective the Korean normal for CDA Referral and Reply Letters and requests by physicians. CIS integrates CDA documents of a nominative patient into one CDA document following the format of CIT. Finally, physicians were surveyed when CIT/CIS adoption, and that they indicated overall satisfaction In Gregorian calendar month 2008, Korea's government launched the National Health info Exchange pilot program.2,3 The selected project web site was national capital National University Bundang Hospital (SNUBH), that may be a tertiary hospital with 1200 beds in conjunction with thirty three connected medical practitioner clinics. within the core of the project, Clinical Document design (CDA) and group action the aid Enterprise Cross- 
Enterprise Document Sharing.b (IHE-XDS.b) were incorporated. CDA, developed by Health Level seven, may be a document markup normal supported XML that specifies the structure and linguistics of "clinical documents" for the aim of exchange and it's a well known normal within the field of health science. 4 Through the primary and second versions free in 2001 and 2005 in sequence,5 numerous comes have adopted CDA for clinical settings.6-8 IHE-XDS.b facilitates depository, maintenance, and exchange of clinical documents.9 A few years when the readying of the Health info Exchange System (HIES) in Choson, however, it became apparent that the increasing quantity of per-patient CDA documents was inflicting many issues. On the HIES, patient care information were scattered in numerous CDA documents, inflicting delay in physician's comprehension of patient's case history. Hospitals in Choson keep financially viable by treating a lot of patients per day as a result of the insurance payment model in Choson is fee-for-service. Thus, physician's time spent with patients per visit is brief in Choson.10 Hence, physicians weren't allowed comfortable time for creating medical selections. Follow-up care service was additionally hindered as a result of insufficient time was allowed. For follow-ups, noticing of abnormal assay results is essential.11 this is often a vital issue as a result of patients with many chronic diseases may even see up to sixteen completely different physicians in an exceedingly year. 12 what is more, referred patients weren't seemingly to tend effective care as a result of an outsized quantity of CDA documents area unit generated anytime a patient was referred and reply created. Therefore, it's imperative that CDA documents be integrated for fast review of patient care history to be given to physicians to confirm quality of care. Unless patient treatment history per clinical item is integrated and classified by written record order, effective care of patients becomes troublesome to realize.

\section{LITERATURE SURVEY}

1 A Prototype Model Using Clinical Document Architecture (CDA) with a Japanese Local Standard : Designing and Implementing a Referral Letter System

In this paper, Since clinical document architecture (CDA) became an American National Standards Institute (ANSI)approved health level seven (HL7) Standard, many countries have begun making an eff ort to make local standards conform to CDA. In order to make CDA compatible with the many diff erent local standards existing in diff erent countries, we designed a prototype model using HL7 CDA R2 with medical markup language (MML).

\section{Application of Portable CDA for Secure Clinical- document Exchange.}

Health Level Seven (HL7) organization published the Clinical Document Architecture (CDA) for exchanging documents among heterogeneous systems and improving medical quality based on the design method in CDA. In practice, although the HL7 organization tried to make medical messages exchangeable, it is still hard to exchange medical messages. There are many issues when two hospitals want to exchange clinical documents, such as patient privacy, network security, budget, and the strategies of the hospital. In this article, we propose a method for the exchange and sharing of clinical documents in an offline model based on the CDA-the Portable CDA. This allows the physician to retrieve the patient's medical record stored in a portal device, but not through the Internet in real time. The security and privacy of CDA data will also be considered..

\section{Clinical Document Architecture integration} system to support patient referral and reply letters. Many Clinical Document Architecture (CDA) referrals and reply documents have been ccumulated for patients since the deployment of the Health Information Exchange System (HIES) in Korea. Clinical data were scattered in many CDA documents and this took too much time for physicians to read. Physicians in Korea spend only limited time per patient as insurances in Korea follow a fee-for-service model. Therefore, physicians were not allowed sufficient time for making medical decisions, and follow-up care service was hindered. To address this, we developed CDA Integration Template (CIT) and CDA Integration System (CIS) for the HIES. The clinical items included in CIT were defined eflecting the Korean Standard for CDA

Referral and Reply Letters and requests by hysicians. CIS integrates CDA documents of a specified patient into a single CDA document following the format of CIT. Finally, physicians were surveyed after CIT/CIS adoption, and they indicated overall satisfaction

\section{PROPOSE SYSTEM}

We describe our CDA document generation and integration Open API service supported cloud computing, through that hospitals ar enabled to handily generate CDA documents while not having to buy proprietary computer code. Our CDA document integration system integrates multiple CDA documents per patient into one CDA document and physicians and patients will browse the clinical knowledge in written record order. The CDA document relating a patient is generated at the clinic wherever the patient is diagnosed. The generated CDA document will be sent to different clinics when patient's consent is noninheritable. The conception of GP doesn't exist in Korean Peninsula, thence it's common for a patient to go to variety of various clinics. The exchange of CDA document is triggered within the following cases: once a doc has to check with the patient's case history; once referral associated reply letters ar required for a patient UN agency is being taken care of by multiple clinics; once the patient is in an emergency and also the medical history has to be reviewed. Our system configuration is that, our system guarantee security of patients knowledge and supply QR code security. Once patient switch one hospital to a different then new hospital scan QR code and obtain patients personal data through QR code.

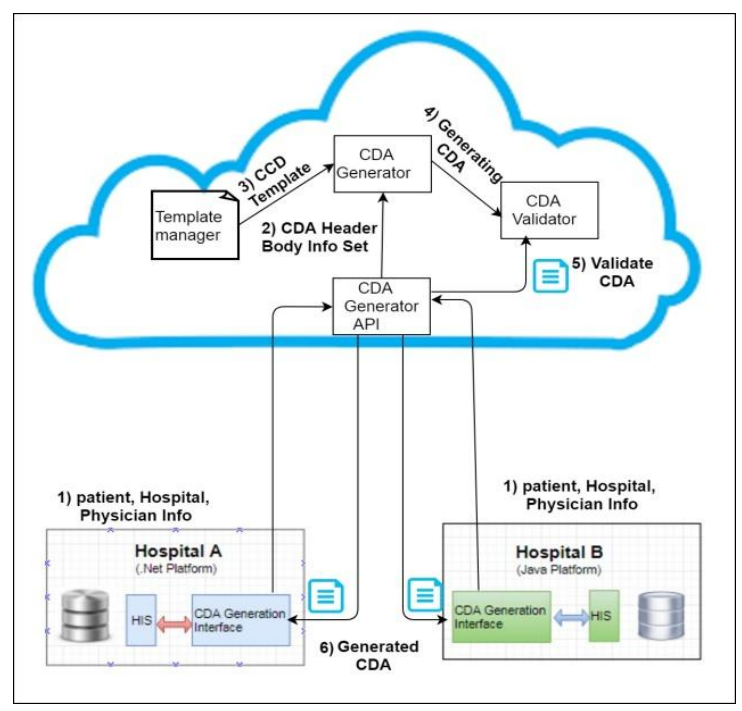

Fig: System Architecture 


\section{ADVANTAGES}

- It helps when the patient is in an emergency and the medical history needs.

- CDA document integration system integrates multiple CDA documents per patient into a single CDA document.

- Interoperability between hospitals help improve patient safety and quality of care.

- $\quad$ Provide QR code security.

\section{CONCLUSION}

Interoperability between hospitals not solely facilitate improve patient safety and quality of care however conjointly scale back time and resources pay on formatting conversion. ability is treated additional necessary because the range of hospitals taking part in hasten will increase if one hospital doesn't support ability, the other hospitals square measure needed to convert formatting of their clinical data to exchange information for hasten. once the amount of hospitals that don't support ability, quality for hasten inevitably increase in proportion. The advantage of API service as ours square measure at the number of resources that hospitals got to assign for ability is merely tokenish. Therefore, providing system that supports ability by counting on a cloud computing platform could also be smart and that we offer the QR code security for patients information that hold on on cloud.

\section{RESULT}

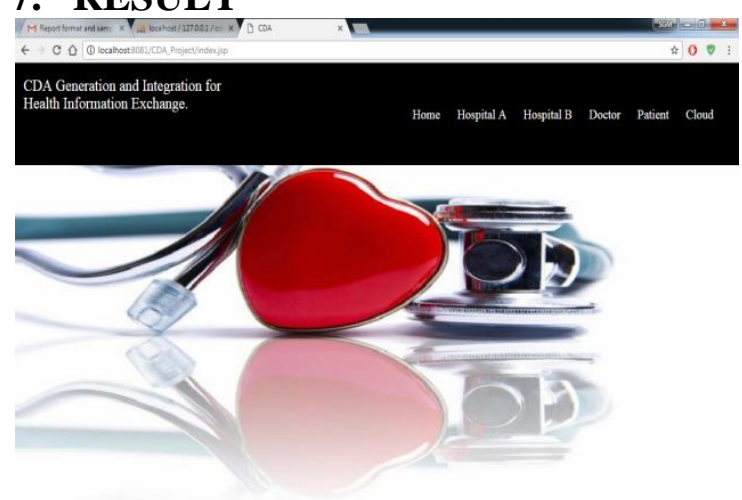

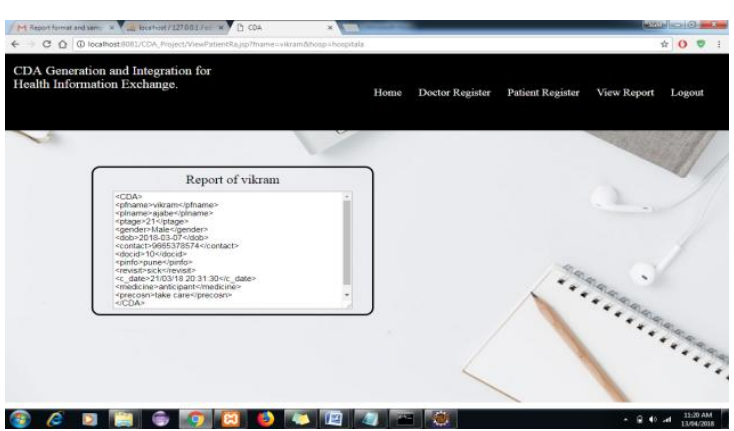

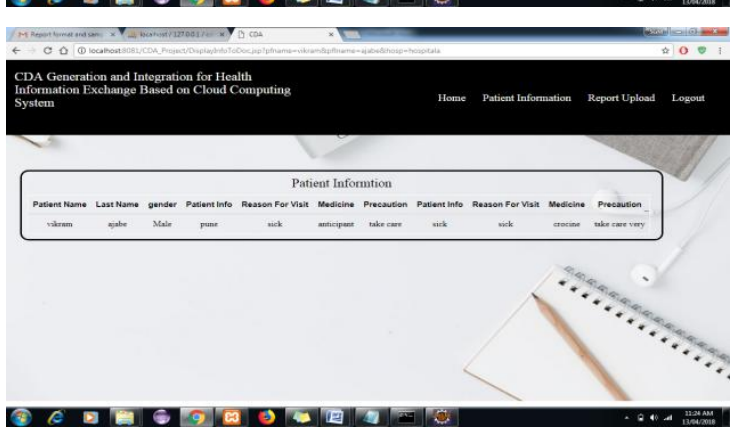

\section{REFERENCES}

[1] K. Huang , Y chang "Application of Portable CDA for Secure Clinical-document Exchange" 2010

[2] H Yong "A prototype model using clinical document architecture (CDA) with a Japanese local standard : designing and implementing a referral letter system"2008

[3] Wen huang , Tai lung Tesung "Generating Standardized Clinical Documents for Medical Information Exchanges" 2010.

[4] S Lee, J song "Clinical document Architecture integration to support patient" 2014

[5] R H Dholin, "The HL7 Clinical documents Architechture” J.Am. Med.Inform.Assoc, Vol.13,no.1,pp.30-39,2006.

[6] K. Huang S. Hsieh,"Application of of portable CDA For secure document exchange",J.Med,Syst.Vol34,pp.531,2010 